\title{
REFINEMENT OF SOME PARTITION IDENTITIES OF MERCA AND YEE
}

\author{
PANKAJ JYOTI MAHANTA AND MANJIL P. SAIKIA
}

\begin{abstract}
Recently, Merca and Yee proved some partition identities involving two new partition statistics. We refine these statistics and generalize the results of Merca and Yee. We also correct a small mistake in a result of Merca and Yee.
\end{abstract}

\section{INTRODUCTION}

A partition of an integer $n$, is a sequence of weakly decreasing positive integers such that they sum up to $n$. The terms of the sequence are called parts and a partition $\lambda$ of $n$ is denoted by $\lambda \vdash n$. We denote by $p(n)$, the number of partitions of $n$. For instance, $2+2+1$ is a partition of 5 and $p(5)=7$. A masterful treatment of this topic is in the book by Andrews And98.

There is a rich history and literature on partitions with various statistics attached to them. Recently, Merca and Yee [MY21] studied several such statistics and proved several interesting results (both analytially and combinatorially). The aim of this paper is to refine the results of Merca and Yee [MY21] by putting in additional constraints on the partition statistics they studied.

The following functions are of interest in this paper.

Definition 1. For a positive integer $n$, we define

(1) $a_{k}(n)$ to be the sum of the parts which are divisible by $k$ counted without multiplicity in all the partitions of $n$,

(2) $a_{k, p}(n)$ to be the sum of the parts which are congruent to $p(\bmod k)$ counted without multiplicity in all the partitions of $n$, where $0 \leq p \leq k-1$, and

(3) $b_{k}(n)$ to be the sum of the distinct parts of $n$ that appear at least $k$ times in all the partitions of $n$.

For example, $a_{3}(5)=6, a_{3,0}(5)=6, a_{3,1}(5)=9, a_{3,2}(5)=11$ and $b_{3}(5)=2$, which can be seen from the fact that the partitions of 5 are

$$
5,4+1,3+2,3+1+1,2+2+1,2+1+1+1,1+1+1+1+1 .
$$

Merca and Yee [MY21] studied related functions. In particular, they studied $a(n)$, the sum of parts counted without multiplicity in all the partitions of $n$ and $b(n)$, the sum of distinct parts that appear at least 2 times in all the partitions of $n$. It is clear from the definition that

$$
a(n)=\sum_{p=0}^{k-1} a_{k, p}(n),
$$

and $b_{2}(n)=b(n)$. So, $a_{k, p}(n)$ and $b_{k}(n)$ can be said to be refinements of $a(n)$ and $b(n)$. They also studied the function $a_{2,0}(n)$ and $a_{2,1}(n)$ which they denoted by $a_{e}(n)$ and $a_{o}(n)$ respectively. We will keep their notation for these special cases in the remainder of this paper.

Merca and Yee [MY21] found the generating functions of $a(n), a_{e}(n), a_{o}(n)$ and $b(n)$, connected these functions in terms of very simple relations and then further connected the function $b(n)$ to

Date: November 23, 2021.

2020 Mathematics Subject Classification. 11P83, 11P84, 05A17, $05 \mathrm{~A} 19$.

Key words and phrases. integer partitions, generating functions, partition identities, truncated partition theorems. 
two other partition functions $M_{\ell}(n)$ and $M P_{\ell}(n)$, which we will define in the next section. The aim of the present paper is to generalize all of these results for our refined functions $a_{k, p}(n)$ and $b_{k}(n)$. While doing this, we also correct a minor error in a result of Merca and Yee [MY21].

The rest of the paper is organized as follows: in Section 2 we state all of our results and show as corollaries all of the results of Merca and Yee [MY21, in Section 3 we prove our results using analytical techniques, in Section 4 we prove all but one of our results using combinatorial techniques, and finally we end the paper with some remarks in Section 5. We closely follow the techniques used by Merca and Yee [MY21] in our proofs.

\section{Results And Corollaries}

We need the notation for the $q$-Pochhammer symbol

$$
(a ; q)_{\infty}=\prod_{n=0}^{\infty}\left(1-a q^{n}\right) \quad \text { for }|q|<1 .
$$

The generating functions for $a_{k}(n), a_{k, p}(n)$ and $b_{k}(n)$ are given in the following theorem.

Theorem 2.1. We have

$$
\begin{aligned}
\sum_{n=1}^{\infty} a_{k}(n) q^{n} & =\frac{1}{(q ; q)_{\infty}} \cdot \frac{k q^{k}}{\left(1-q^{k}\right)^{2}} \\
\sum_{n=1}^{\infty} a_{k, p}(n) q^{n} & =\frac{1}{(q ; q)_{\infty}} \cdot \frac{\left(p q^{p-k}+(k-p) q^{p}\right) q^{k}}{\left(1-q^{k}\right)^{2}} \\
\sum_{n=1}^{\infty} b_{k}(n) q^{n} & =\frac{1}{(q ; q)_{\infty}} \cdot \frac{q^{k}}{\left(1-q^{k}\right)^{2}} .
\end{aligned}
$$

From the above theorem (as well as combinatorially, which we will prove later) the following result follows.

Theorem 2.2. For all $n \geq 1$, we have

(1) $a_{k}(n)=k b_{k}(n)$, and

(2) $a_{k, p}(n)=(k-p) b_{k}(n-p)+p b_{k}(n+k-p)$.

As easy corollaries of the above results, two results of Merca and Yee [MY21] follow.

Corollary 2.3 (Theorem 1.2, MY21]). We have

$$
\begin{aligned}
& \sum_{n=1}^{\infty} a_{e}(n) q^{n}=\sum_{n=1}^{\infty} a_{2,0}(n) q^{n}=\frac{1}{(q ; q)_{\infty}} \cdot \frac{2 q^{2}}{\left(1-q^{2}\right)^{2}}, \\
& \sum_{n=1}^{\infty} a_{o}(n) q^{n}=\sum_{n=1}^{\infty} a_{2,1}(n) q^{n}=\frac{1}{(q ; q)_{\infty}} \cdot \frac{q\left(1+q^{2}\right)}{\left(1-q^{2}\right)^{2}},
\end{aligned}
$$

and

$$
\sum_{n=1}^{\infty} a(n) q^{n}=\frac{1}{(q ; q)_{\infty}} \cdot \frac{q}{(1-q)^{2}} .
$$

Corollary 2.4 (Theorem 1.3, MY21]). For all $n \geq 1$, we have

(1) $a_{e}(n)=a_{2,0}(n)=2 b(n)$,

(2) $a_{o}(n)=a_{2,1}(n)=b(n+1)+b(n-1)$, and

(3) $a(n)=a_{2,0}(n)+a_{2,1}(n)=b(n+1)+2 b(n)+b(n-1)$. 
Andrews and Merca AM12 introduced a new partition function $M_{\ell}(n)$, which counts the number of partitions of $n$ where $\ell$ is the least positive integer that is not a part and there are more parts which are greater than $\ell$ than there are parts less than $\ell$. For instance $M_{3}(5)=0$. We can connect this function with $b_{k}(n)$ in the following way.

Theorem 2.5. For any positive integer $k, \ell$ and $n$, we have

$$
\begin{array}{r}
(-1)^{\ell-1}\left(\sum_{j=-(\ell-1)}^{\ell}(-1)^{j} b_{k}(n-j(3 j-1) / 2)-\frac{\left.1+(-1)^{[n \equiv 0}(\bmod k)\right]+1}{2} \cdot \frac{n}{k}\right) \\
=\sum_{j=1}^{\lfloor n / k\rfloor} j M_{\ell}(n-k j),
\end{array}
$$

where we have used the Iverson bracket, $[P]$ which returns the value 1 if the logical proposition $P$ is satisfied, and returns 0 otherwise.

The following are two easy corollaries of the above theorem.

Corollary 2.6. For any positive integer $k, \ell$ and $n$, we have

$$
(-1)^{\ell-1}\left(\sum_{j=-(\ell-1)}^{\ell}(-1)^{j} b_{k}(n-j(3 j-1) / 2)-\frac{1+(-1)^{[n \equiv 0 \quad(\bmod k)]+1}}{2} \cdot \frac{n}{k}\right) \geq 0 .
$$

Corollary 2.7. For any positive integer $k$ and $n$, we have

$$
\sum_{j=-\infty}^{\infty}(-1)^{j} b_{k}(n-j(3 j-1) / 2)=\frac{1+(-1)^{[n \equiv 0 \quad(\bmod k)]+1}}{2} \cdot \frac{n}{k} .
$$

From the above theorem and corollaries, the following results follow easily.

Corollary 2.8 (Theorem 1.4, [MY21]). For any positive integers $\ell$ and $n$, we have

$$
(-1)^{\ell-1}\left(\sum_{j=-(\ell-1)}^{\ell}(-1)^{j} b(n-j(3 j-1) / 2)-\frac{1+(-1)^{n}}{2} \cdot \frac{n}{2}\right)=\sum_{j=1}^{\lfloor n / 2\rfloor} j M_{\ell}(n-2 j) .
$$

Corollary 2.9 (Corollary 1.5, [MY21]). For any positive integers $\ell$ and $n$, we have

$$
(-1)^{\ell-1}\left(\sum_{j=-(\ell-1)}^{\ell}(-1)^{j} b(n-j(3 j-1) / 2)-\frac{1+(-1)^{n}}{2} \cdot \frac{n}{2}\right) \geq 0 .
$$

Corollary 2.10 (Corollary 1.6, [MY21]). For any positive integer n, we have

$$
\sum_{j=-\infty}^{\infty}(-1)^{j} b(n-j(3 j-1) / 2)=\frac{1+(-1)^{n}}{2} \cdot \frac{n}{2} .
$$

Andrews and Merca AM18] studied a new partition function $M P_{\ell}(n)$, which counts the number of partitions of $n$ in which the first part larger than $2 k-1$ is odd and appears exactly $k$ times, and all other parts appear at most one time. For instance, $M P_{3}(5)=3$. We can connect the function $b_{k}(n)$ with $M P_{\ell}(n)$ using a new function $c_{k}(n)$ in the following way. 
Theorem 2.11. For any positive integer $k, \ell$ and $n$, we have

$$
\begin{array}{r}
(-1)^{\ell-1}\left(\sum_{j=0}^{2 \ell-1}(-1)^{\frac{j(j+1)}{2}} b_{k}(n-j(j+1) / 2)-\frac{\left.1+(-1)^{[n \equiv 0}(\bmod k)\right]+1}{2} \cdot c_{k}(n)\right) \\
=\sum_{j=0}^{n} c_{k}(j) M P_{\ell}(n-j),
\end{array}
$$

where we have used the Iverson bracket, $[P]$ which returns the value 1 if the logical proposition $P$ is satisfied, and returns 0 otherwise, and the function $c_{k}(n)$ is defined as

$$
c_{k}(n)=\sum_{j=1}^{\lfloor n / k\rfloor} j Q\left(\frac{n-k j}{2}\right)
$$

and $Q(m)$ denotes the number of partitions of $m$ into distinct parts. Here $Q(x)=0$ if $x \notin \mathbb{N}$.

The following are two easy corollaries of the above theorem.

Corollary 2.12. For any positive integers $k, \ell$ and $n$, we have

$$
(-1)^{\ell-1}\left(\sum_{j=0}^{2 \ell-1}(-1)^{\frac{j(j+1)}{2}} b_{k}(n-j(j+1) / 2)-\frac{1+(-1)^{[n \equiv 0 \quad(\bmod k)]+1}}{2} \cdot c_{k}(n)\right) \geq 0 .
$$

Corollary 2.13. For positive integers $n$ and $k$, we have

$$
\sum_{j=0}^{\infty}(-1)^{\frac{j(j+1)}{2}} b_{k}(n-j(j+1) / 2)=\frac{\left.1+(-1)^{[n \equiv 0}(\bmod k)\right]+1}{2} \cdot c_{k}(n)
$$

From the above theorem and corollaries, the following results of Merca and Yee [MY21] follow as corollaries. Here we have corrected the exponent of the -1 inside the summation in the left hand side, which is $\frac{j(j+1)}{2}$, but was mentioned as $j$ by Merca and Yee [MY21].

Corollary 2.14 (Theorem 1.7, [MY21]). For any positive integer $\ell$ and $n$, we have

$$
(-1)^{\ell-1}\left(\sum_{j=0}^{2 \ell-1}(-1)^{\frac{j(j+1)}{2}} b(n-j(j+1) / 2)-\frac{1+(-1)^{n}}{2} \cdot c\left(\frac{n}{2}\right)\right)=\sum_{j=1}^{\lfloor n / 2\rfloor} c(j) M P_{\ell}(n-2 j),
$$

where $c(n)$ is the number of subsets of $\{1,2, \ldots, n\}$ which contains a number that is greater than the sum of the other numbers in the subset.

Proof. We notice that

$$
c_{2}(2 n)=\sum_{j=1}^{n} j Q(n-j)=\sum_{m=0}^{n-1}(n-m) Q(m),
$$

which was shown to be equal to $c(n)$ in the proof of Theorem 4.1 in Merca and Yee's [MY21] work. So, we have $c_{2}(n)=c\left(\frac{n}{2}\right)$. Putting $k=2$ in Theorem 2.11 we get the result.

Corollary 2.15 (Corollary 4.2, [MY21]). Let $\ell$ and $n$ be positive integers, then we have

$$
(-1)^{\ell-1}\left(\sum_{j=0}^{2 \ell-1}(-1)^{\frac{j(j+1)}{2}} b(n-j(j+1) / 2)-\frac{1+(-1)^{n}}{2} \cdot c\left(\frac{n}{2}\right)\right) \geq 0 .
$$

Corollary 2.16 (Corollary 4.3, [MY21]). Let $n$ be a positive integer, then we have

$$
\sum_{j=0}^{\infty}(-1)^{\frac{j(j+1)}{2}} b(n-j(j+1) / 2)=\frac{1+(-1)^{n}}{2} \cdot c\left(\frac{n}{2}\right) .
$$




\section{Analytical Proofs of our Main Results}

In this section, we prove all the theorems stated in the previous section, using analytical methods. Our proofs follow closely the techniques used by Merca and Yee [MY21].

3.1. Proof of Theorems 2.1 and 2.2. We start with the generating function for partitions where the power of $z$ keeps track of parts with multiplicity $\geq k$,

$$
\begin{aligned}
\prod_{j=1}^{\infty}\left(1+q^{j}+q^{2 j}+\cdots+q^{(k-1) j}+\right. & \left.z^{j}\left(q^{k j}+q^{(k+1) j}+\cdots\right)\right) \\
& =\prod_{j=1}^{\infty}\left(\frac{q^{k j}-1}{q^{j}-1}+z^{j} \frac{q^{k j}}{1-q^{j}}\right)=\frac{1}{(q ; q)_{\infty}} \prod_{j=1}^{\infty}\left(1+\left(z^{j}-1\right) q^{k j}\right) .
\end{aligned}
$$

Now, taking the derivative w.r.t. $z$ and setting $z \rightarrow 1$ we get,

$$
\sum_{n=1}^{\infty} b_{k}(n) q^{n}=\frac{1}{(q ; q)_{\infty}} \sum_{j=1}^{\infty} j q^{k j}=\frac{1}{(q ; q)_{\infty}} \cdot \frac{q^{k}}{\left(1-q^{k}\right)^{2}} .
$$

In a similar way, we have

$$
\begin{aligned}
& \sum_{n=1}^{\infty} a_{k, p}(n) q^{n} \\
& =\frac{\partial}{\partial z}\left(1-q^{p}+z^{p} q^{p}\right) \prod_{j=1}^{\infty}\left(\left(1+q^{j}+q^{2 j}+\cdots\right)-\left(q^{k j+p}+q^{(k+1) j+p}+\cdots\right)\right. \\
& \left.+z^{k j+p}\left(q^{k j+p}+q^{(k+1) j+p}+\cdots\right)\right)\left.\right|_{z=1} .
\end{aligned}
$$

We subtract $\left(q^{k j+p}+q^{(k+1) j+p}+\cdots\right)$ from the first term, because we count the parts of the type $k j+p$ where $j \geq 1$ in the third term, and we multiply by $\left(1-q^{p}+z^{p} q^{p}\right)$ because of the parts of the form $k j+p$ where $j=0$. Therefore,

$$
\begin{aligned}
\sum_{n=1}^{\infty} a_{k, p}(n) q^{n} & =\left.\frac{\partial}{\partial z}\left(1-q^{p}+z^{p} q^{p}\right) \prod_{j=1}^{\infty}\left(\frac{1-q^{k j+p}}{1-q^{j}}+z^{k j+p} \frac{q^{k j+p}}{1-q^{j}}\right)\right|_{z=1} \\
& =\left.\frac{1}{(q ; q)_{\infty}} \frac{\partial}{\partial z} \prod_{j=0}^{\infty}\left(1+\left(z^{k j+p}-1\right) q^{k j+p}\right)\right|_{z=1} \\
& =\frac{1}{(q ; q)_{\infty}} \sum_{j=0}^{\infty}(k j+p) q^{k j+p} \\
& =\frac{1}{(q ; q)_{\infty}}\left(k q^{p} \frac{q^{k}}{\left(1-q^{k}\right)^{2}}+p q^{p} \frac{1}{1-q^{k}}\right) \\
& =\frac{1}{(q ; q)_{\infty}} \cdot \frac{p q^{p}+(k-p) q^{p+k}}{\left(1-q^{k}\right)^{2}} .
\end{aligned}
$$

The case $p=0$ in the above will give us the generating function for $a_{k}(n)$.

Theorem 2.2 immediately follows from Theorem 2.1; we just compare coefficients.

3.2. Proof of Theorem 2.5. The generating function for $M_{\ell}(n)$ was found by Andrews and Merca [AM12, when they studied a truncated version of Euler's pentagonal number theorem

$$
\frac{(-1)^{\ell-1}}{(q ; q)_{\infty}} \sum_{n=-(\ell-1)}^{\ell}(-1)^{n} q^{n(3 n-1) / 2}=(-1)^{\ell-1}+\sum_{n=\ell}^{\infty} \frac{q^{\left(\begin{array}{l}
\ell \\
2
\end{array}\right)+(\ell+1) n}}{(q ; q)_{n}}\left[\begin{array}{c}
n-1 \\
\ell-1
\end{array}\right],
$$


where $\ell \geq 1,(a ; q)_{n}=\frac{(a ; q)_{\infty}}{\left(a q^{n} ; q\right)_{\infty}}$ and the Gausssian binomial $\left[\begin{array}{c}n \\ \ell\end{array}\right]$ equals $\frac{(q ; q)_{n}}{(q ; q)_{\ell}(q ; q)_{n-\ell}}$ whenever $0 \leq \ell \leq n$ and is 0 otherwise. The sum on the right hand side of equation (13.1) is the generating function of $M_{\ell}(n)$, that is

$$
\sum_{n=0}^{\infty} M_{\ell}(n) q^{n}=\sum_{n=\ell}^{\infty} \frac{q^{\left(\begin{array}{l}
\ell \\
2
\end{array}\right)+(\ell+1) n}}{(q ; q)_{n}}\left[\begin{array}{l}
n-1 \\
\ell-1
\end{array}\right]
$$

We now multiply both sides of equation (3.1) by

$$
\sum_{n=0}^{\infty} n q^{k n}=\frac{q^{k}}{\left(1-q^{k}\right)^{2}}
$$

which gives us (after using equation (3.2) ),

$$
\begin{aligned}
(-1)^{\ell-1}\left(\left(\sum_{n=1}^{\infty} b_{k}(n) q^{n}\right)\left(\sum_{n=-(\ell-1)}^{\ell}(-1)^{n} q^{n(3 n-1) / 2}\right)-\sum_{n=0}^{\infty} n q^{k n}\right) & =\left(\sum_{n=0}^{\infty} n q^{k n}\right)\left(\sum_{n=0}^{\infty} M_{\ell}(n) q^{n}\right) .
\end{aligned}
$$

Using the Cauchy product of two power series, Theorem 2.5 follows from the above.

3.3. Proof of Theorem 2.11. The generating function for $M P_{\ell}(n)$ was found by Andrews and Merca [AM18] when they considered a truncated theta identify of Gauss,

$$
\frac{\left(-q ; q^{2}\right)_{\infty}}{\left(q^{2} ; q^{2}\right)_{\infty}} \sum_{j=0}^{2 \ell-1}(-q)^{j(j+1) / 2}=1+(-1)^{\ell-1} \frac{\left(-q ; q^{2}\right)_{\ell}}{\left(q^{2} ; q^{2}\right)_{\ell-1}} \sum_{j=0}^{\infty} \frac{q^{\ell(2 \ell+2 j+1)}\left(-q^{2 \ell+2 j+3} ; q^{2}\right)_{\infty}}{\left(q^{2 \ell+2 j+2} ; q^{2}\right)_{\infty}}
$$

The sum on the right hand side of equation (3.3) is the generating function of $M P_{\ell}(n)$.

We now multiply both sides of equation (3.3) by $\frac{q^{k}}{\left(1-q^{k}\right)^{2}} \cdot\left(-q^{2} ; q^{2}\right)_{\infty}$ and deduce the following identity

$$
\begin{aligned}
(-1)^{\ell-1}\left(\left(\sum_{n=0}^{\infty} b_{k}(n) q^{n}\right)\left(\sum_{n=0}^{2 \ell-1}(-q)^{n(n+1) / 2}\right)\right. & \left.-\frac{q^{k}}{\left(1-q^{k}\right)^{2}} \cdot\left(-q^{2} ; q^{2}\right)_{\infty}\right) \\
& =\left(\frac{q^{k}}{\left(1-q^{k}\right)^{2}} \cdot\left(-q^{2} ; q^{2}\right)_{\infty}\right)\left(\sum_{n=0}^{\infty} M P_{\ell}(n) q^{n}\right) .
\end{aligned}
$$

We know that the generating function of the number of partitions into distinct parts is

$$
\sum_{n=0}^{\infty} Q(n) q^{n}=(-q ; q)_{\infty}
$$


Using this, we have

$$
\begin{aligned}
\frac{q^{k}}{\left(1-q^{k}\right)^{2}} \cdot\left(-q^{2} ; q^{2}\right)_{\infty} & =\sum_{n=0}^{\infty} n q^{k n} \cdot \sum_{m=0}^{\infty} Q(m) q^{2 m} \\
& =\sum_{n=0}^{\infty} \sum_{j=1}^{\lfloor n / k\rfloor} j Q\left(\frac{n-k j}{2}\right) q^{n} \\
& =\sum_{n=0}^{\infty} c_{k}(n) q^{n} .
\end{aligned}
$$

Putting this in equation (3.4) we get,

$$
\begin{aligned}
(-1)^{\ell-1}\left(\left(\sum_{n=0}^{\infty} b_{k}(n) q^{n}\right)\left(\sum_{n=0}^{2 \ell-1}(-q)^{n(n+1) / 2}\right)-\sum_{n=0}^{\infty} c_{k}(n) q^{n}\right) & =\left(\sum_{n=0}^{\infty} c_{k}(n) q^{n}\right)\left(\sum_{n=0}^{\infty} M P_{\ell}(n) q^{n}\right) .
\end{aligned}
$$

Using the Cauchy product of two power series, Theorem 2.11 follows from the above.

\section{Combinatorial Proofs of some of our Results}

In this section we give combinatorial proofs of all but one (Theorem 2.11) of our results. The approach again closely follows that of Merca and Yee [MY21].

4.1. Proof of Theorem 2.2. For part (1), we note that for a partition $\lambda \vdash n$, if $k a$ is a part of $\lambda$ then we split this part into $k$ a's while keeping the remaining parts of $\lambda$ unchanged. Let us call the new partition $\mu$, then clearly the part $a$ has multiplicity at least $k$ in $\mu$, so we get

$$
\begin{aligned}
a_{k}(n) & =\sum_{\lambda \vdash n} \text { different parts divisble by } k \\
& =k \sum_{\mu \vdash n} \text { differents parts with multiplicity } \geq k=k b_{k}(n) .
\end{aligned}
$$

For part (2), let $k a+p$ be a part of $\lambda \vdash n$ which is counted in $a_{k, p}(n)$. We now split $k a$ into $k a$ 's while keeping the remaining parts unchanged to get a new partition $\mu \vdash n-p$. Again, we split $(k a+p)+(k-p)$ into $k(a+1)$ 's while keeping the remaining parts unchanged to get a new partition $\nu \vdash n+k-p$. We have

$$
\begin{aligned}
a_{k, p}(n)= & \sum_{\lambda \vdash n} \text { different parts } \equiv p \quad(\bmod k) \\
= & (k-p) \sum_{\mu \vdash n-p} \text { different parts with multiplicity } \geq k \\
& +p \sum_{\nu \vdash n+k-p} \text { different parts with multiplicity } \geq k \\
= & (k-p) b_{k}(n-p)+p b_{k}(n+k-p) .
\end{aligned}
$$

4.2. Proof of Theorem 2.1. We prove the generating function for $a_{k}(n)$ here; the other two generating functions can be proved combinatorially by combining the previous subsection with this proof. In fact, our proof is the same when 2 is replaced by $k$ in the proof of Corollary 2.3 given by Merca and Yee [MY21], so for the sake of brevity we just outline the steps.

We work with two sets of overpartitions, let $\bar{P}_{k}(n)$ be the set of overpartitions of $n$ where exactly one part divisible by $k$ is overlined, and let $\bar{A}_{k}(n)$ be the set of colored overpartitions of 
$n$ where exactly one part divisible by $k$ is overlined and at most one other part divisible by $k$ is colored with blue color. For instance, we have

$$
\bar{P}_{3}(6)=\{\overline{6}, \overline{3}+3, \overline{3}+2+1, \overline{3}+1+1+1\},
$$

and

$$
\bar{A}_{3}(6)=\{\overline{6}, \overline{3}+3, \overline{3}+3, \overline{3}+2+1, \overline{3}+1+1+1\} .
$$

Clearly, $\bar{P}_{k}(n)$ is a subset of $\bar{A}_{k}(n)$, and we have

$$
a_{k}(n)=\sum_{\lambda \in \bar{P}_{k}(n)} \text { the overlined part of } \lambda \text {. }
$$

Also note that for each partition in $\bar{A}_{k}(n)$ we can decompose it into a tuple $(\lambda, \mu, \nu)$ where $\lambda$ is the overline part, $\mu$ is the colored part and $\nu$ are the non-colored parts. This gives us

$$
\sum_{n \geq 0} \bar{A}_{k}(n) q^{n}=\frac{q^{k}}{\left(1-q^{k}\right)} \cdot \frac{1}{\left(1-q^{k}\right)} \cdot \frac{1}{(q ; q)_{\infty}} .
$$

We now set up the following surjection from $\bar{A}_{k}(n)$ to $\bar{P}_{k}(n)$ : if there is a colored part, we merge it with the overlined part to get a resulting overlined part. The new partition is clearly in $\bar{P}_{k}(n)$, and if there are no colored parts then we keep the partition unchanged. Now, for an overlined part $\overline{k a}$ of $\mu \in \bar{P}_{k}(n)$, there are $a$ ways to merge an overlined part with a colored part to get $k a$, so we have

$$
\sum_{\mu \in \bar{P}_{k}(n)} \text { the overline part of } \mu=\sum_{\nu \in \bar{A}_{k}(n)} k .
$$

From equations (4.1), (4.2) and (4.3) we get

$$
\sum_{n \geq 0} a_{k}(n) q^{n}=\frac{1}{(q ; q)_{\infty}} \cdot \frac{k q^{k}}{\left(1-q^{k}\right)^{2}}
$$

4.3. Proof of Theorem 2.5. Again, our proof is similar to the proof of Corollary 2.6, given by Merca and Yee [MY21], so we mention the main steps without going into too much details. Theorem 2.5 is equivalent to the following

$$
\begin{array}{r}
(-1)^{\ell-1}\left(\sum_{j=-(\ell-1)}^{\ell}(-1)^{j}\left(\sum_{\lambda \in \bar{P}_{k}(n-j(3 j-1) / 2} \text { overlined part of } \lambda\right)\right. \\
\left.\quad-\frac{\left.1+(-1)^{[n \equiv 0}(\bmod k)\right]+1}{2} \cdot n\right)=\sum_{j=1}^{\lfloor n / k\rfloor} k j M_{\ell}(n-k j),
\end{array}
$$

where we have used Theorem 2.2 and equation (4.1).

We note that

$$
\sum_{\lambda \in \bar{P}_{k}(n)} \text { overlined part of } \lambda=\sum_{m=1}^{\lfloor n / k\rfloor} k m \sum_{\mu \vdash(n-k m)} 1=\sum_{m=1}^{\lfloor n / k\rfloor} k m \cdot p(n-k m) .
$$

The above equation is true since any partition $\lambda \in \bar{P}_{k}(n)$ can be made into a pair of partitions $(\nu, \mu)$ where $\nu$ is the overlined part and $\mu$ is then an ordinary partition. 
So, we get

$$
\begin{aligned}
(-1)^{\ell-1} \sum_{j=-(\ell-1)}^{\ell}(-1)^{j} & \left(\sum_{\lambda \in \bar{P}_{k}(n-j(3 j-1) / 2} \text { overlined part of } \lambda\right) \\
& =(-1)^{\ell-1} \sum_{j=-(\ell-1)}^{\ell}(-1)^{j} \sum_{m=1}^{\lfloor(n-j(3 j-1) / 2) / 2\rfloor} k m \cdot p(n-j(3 j-1) / 2-k m) .
\end{aligned}
$$

The above is equivalent to

$$
\sum_{m=1}^{\lfloor n / k\rfloor} k m\left((-1)^{\ell-1} \sum_{j=-(\ell-1)}^{\ell}(-1)^{j} p(n-k m-j(3 j-1) / 2)\right)
$$

where we rearrange the summation and take $p(n)=0$ if $n<0$.

Merca and Yee [MY21] have given a combinatorial proof of the truncated pentagonal number theorem, which is equivalent to the following identity

$$
\left.(-1)^{\ell-1} \sum_{j=0}^{\ell-1}\right)(-1)^{j}(p(n-j(3 j+1) / 2)-p(n-(j+1)(3 j+2) / 2))=M_{\ell}(n) .
$$

Using equation (4.5) in (4.4), we get that (4.4) is equal to

$$
\sum_{m=1}^{\lfloor n / k\rfloor} k m \cdot M_{\ell}(n-k m)+(-1)^{\ell-1} \cdot \frac{1+(-1)^{[n \equiv 0 \quad(\bmod k)]+1}}{2} \cdot n,
$$

which proves the result, since equation (4.5) already has a combinatorial proof. We need the term $(-1)^{\ell-1} n$ when $n \equiv 0(\bmod k)$ because, if $n=k r$ for some $r$ and $m=n / k$, then without this term we get

$$
(-1)^{\ell-1} n p(0)=n M_{k}(0) \Rightarrow n=0 .
$$

\section{Concluding Remarks}

(1) A combinatorial proof of Theorem 2.11 is left as an open problem. Any combinatorial proof of Theorem 2.11 would hinge on a combinatorial interpretation of $c_{k}(n)$, like we have for $c_{2}(2 n)$. So, a first step towards a combinatorial proof would be such an interpretation of $c_{k}(n)$.

(2) Identities of the type in Theorems 2.5 and 2.11 are also known for some other partition statistics, for instance one can see some recent work of Merca [Mer20]. It would be interesting to see if one can relate such partition statistics with the ones introduced in this paper.

\section{ACKNOWLEDGEMENTS}

The second author is partially supported by the Leverhulme Trust Research Project Grant RPG-2019-083. The authors thank Dr. Nilufar Mana Begum for bringing the paper of Merca and Yee [MY21] to their notice, and Prof. Nayandeep Deka Baruah for encouragement. The authors also thank the editor and the anonymous referee for helpful comments. 


\section{REFERENCES}

[AM12] George E. Andrews and Mircea Merca. The truncated pentagonal number theorem. J. Combin. Theory Ser. A, 119(8):1639-1643, 2012.

[AM18] George E. Andrews and Mircea Merca. Truncated theta series and a problem of Guo and Zeng. J. Combin. Theory Ser. A, 154:610-619, 2018.

[And98] George E. Andrews. The theory of partitions. Cambridge Mathematical Library. Cambridge University Press, Cambridge, 1998. Reprint of the 1976 original.

[Mer20] Mircea Merca. The powers of two as sums over partitions. Quaest. Math., to appear, 2020.

[MY21] Mircea Merca and Ae Ja Yee. On the sum of parts with multiplicity at least 2 in all the partitions of $n$. Int. J. Number Theory, 17(3):665-681, 2021.

Gonit Sora, Dhalpur, Assam 784165, India

Email address: pankaj@gonitsora.com

School of Mathematics, Cardiff University, Cardiff, CF24 4AG, UK

Email address: manjil@saikia.in 